\title{
Bilateral carcinomas of the breast with local recurrence: analysis of genetic relationship of the tumors
}

\author{
Peter Regitnig ${ }^{1}$, Ferdinand Ploner $^{2}$, Martina Maderbacher ${ }^{1}$ and Sigurd F Lax ${ }^{1}$ \\ ${ }^{1}$ Department of Pathology and ${ }^{2}$ Department of Internal Medicine, Division of Medical Oncology, Medical \\ University of Graz, Graz, Austria
}

\begin{abstract}
Local recurrence of bilateral breast carcinomas is rare, but of biological interest, since it is unclear as to which tumor the local recurrence is related to, the ipsilateral or the contralateral, or whether it is an independent neoplasm. The aim of this study was to investigate the genetic relationship of bilateral breast carcinomas to each other and to their local recurrences. Eight cases of bilateral breast carcinomas, five with and three without local recurrence were analyzed using a microsatellite assay for 13 microsatellite loci. The presence of loss of heterozygosity and microsatellite instability in the various tumors was used for clonal analysis. All eight bilateral breast carcinomas showed divergent alterations in at least two microsatellite loci, which ruled out a genetic relationship. Four of five local recurrences were genetically related to the ipsilateral tumor and unrelated to the contralateral tumor. Only one local recurrence that occurred 11.8 years after the surgery of an infiltrative lobular carcinoma simultaneously with distant metastases was genetically related to the contralateral breast carcinoma. Although the number of cases in our study is limited, there is evidence that local recurrence of bilateral breast carcinoma frequently arises from the ipsilateral tumor.
\end{abstract}

Modern Pathology (2004) 17, 597-602, advance online publication, 12 March 2004; doi:10.1038/modpathol.3800089

Keywords: bilateral breast carcinoma; local recurrence; microsatellite analysis; loss of heterozygosity; clonality

Epidemiologic data on breast carcinomas show that $2-11 \%$ of breast carcinoma patients will develop contralateral breast carcinoma in their lifetime. Women with unilateral breast carcinoma are at a 2-6-fold increased risk of developing contralateral breast carcinoma, compared to the risk in the general population. ${ }^{1}$

Bilateral breast carcinomas are of particular interest with respect to their relationship, whether they represent independent neoplasms or occur by metastatic tumor spread. This issue becomes more complex in the setting of local tumor recurrence. In this situation, the question arises whether the local recurrence is related to one of the bilateral tumors or is an independent, de novo arising neoplasm, or whether both of the tumors and the recurrence are metastases of the other tumor. The fact that whether bilateral breast carcinomas and its local recurrence have to be considered a local or a

Correspondence: Dr SF Lax, MD, Department of Pathology, General Hospital Graz West, Goestinger strasse 22, Graz A-8020, Austria.

E-mail: sigurd.lax@uni-graz.at

Received 14 August 2003; revised 24 November 2003; accepted 5 December 2003; published online 12 March 2004 metastatic process has important therapeutic and prognostic implications.

A second independently arising breast carcinoma is favored, if the histological tumor types are different. However, if the histological tumor types are identical, the distinction between a subsequently occurred novel tumor and a local recurrence of the original tumor is difficult. Conventional parameters such as the histopathological grade and various immunohistochemical analyses such as estrogen, progesterone and HER-2/neu receptor expression are not reliable to prove the relationship of these tumors.

During recent years, several studies using different molecular methods have indicated that bilateral breast carcinomas are most likely genetically unrelated, ${ }^{2-4}$ irrespective of synchronous or metachronous occurrence. These findings suggest that bilateral carcinomas of the breast arise independently and not due to metastatic spread. This hypothesis is supported by the fact that patients with bilateral carcinomas have a better prognosis compared to patients with distant metastases. ${ }^{5}$ However, the relationship of a local recurrence to either of the bilateral tumors has not yet been studied by molecular genetic analysis. 
The aim of this study was to assess the genetic relationship of unilateral local recurrences in the setting of bilateral breast carcinoma by microsatellite analysis on formalin-fixed, paraffin-embedded tissue of the primary and the locally recurrent tumors. We are aware that the number of cases in this study is small due to a limited availability of appropriate cases but, nevertheless, we consider the results of common interest.

\section{Materials and methods}

\section{Patient Selection}

The cases were retrieved from the files of the Department of Pathology, University of Graz, Austria. Although at this department, nearly 900 breast carcinoma diagnoses are made each year, only five patients matched the search criteria. A local recurrence (LR) was defined as recurrent carcinoma within the same breast after breast-conserving therapy or within the ipsilateral mammary region after mastectomy. Tumor type and histopathological grade were reassessed for tumors and recurrences according to standardized guidelines. ${ }^{6,7}$ Postoperative staging followed the UICC guidelines. ${ }^{8}$

\section{Molecular Analysis}

Paraffin blocks with representative primary and recurrent tumor tissue, which had been fixed in $8 \%$ neutral buffered formalin, were selected from each case. Tumor-free lymph nodes were used as paired normal (non-neoplastic) tissue controls.

Tissue sections, $5 \mu \mathrm{m}$ thick, were deparaffinized in xylene and stained with hematoxylin. To obtain at least $70 \%$ of pure tumor DNA and to avoid contamination of normal tissue by tumor cells, all tissues were microdissected using an inverted microscope and sterile blades and needles. The microdissected tissue samples were incubated in xylene for $15 \mathrm{~min}$ at room temperature, centrifuged at $13000 \mathrm{rpm}$. and washed twice with $100 \%$ ethanol. The pellets were dried in a vacuum centrifuge for $30 \mathrm{~min}$ at room temperature and resuspended in 100-300 $\mu \mathrm{l}$ of $50 \mathrm{mmol} / \mathrm{l}$ Tris buffer (pH 9.0) containing $400 \mathrm{ng} / \mu \mathrm{l}$ proteinase $\mathrm{K}$ (Boehringer Mannheim, Germany) overnight at $55^{\circ} \mathrm{C}$.

For microsatellite analysis, 13 microsatellites were used. The microsatellites (Table 1) were selected according to locations of known tumor suppressor genes and microsatellite loci, respectively, involved in breast carcinoma development and progression (D3S1244, ${ }^{9}$ D10S215, D11S528, D17S855, ${ }^{10}$ D17S250, ${ }^{11}$ D18S59 ${ }^{12}$ D10S2491 ${ }^{13}$ and

Table 1 Microsatellites used in this study including localization within the human genome, their relation to known genes and specific PCR details

\begin{tabular}{|c|c|c|c|c|c|}
\hline $\begin{array}{l}\text { Microsatel- } \\
\text { lite locus }\end{array}$ & Chromosome & Related gene (location to gene) & Primer sequence (forward / reverse) & $\begin{array}{c}\text { PCR } \\
\text { buffer }{ }^{a}\end{array}$ & $\begin{array}{c}\text { Annealing } \\
\text { temperature } \\
\left({ }^{\circ} \mathrm{C}\right)\end{array}$ \\
\hline BAT 26 & $2 \mathrm{p} 16$ & MSH2 (intragenic) & $\begin{array}{l}\text { TGACTACTTTTGACTTCAGCC } \\
\text { AACCATTCAACATTTTTAACCC }\end{array}$ & $\mathrm{E}$ & 54 \\
\hline D3S1244 & 3pter-24.2 & Unknown & $\begin{array}{l}\text { GTGCCCTTCCAGGAGTT } \\
\text { AGTGAGGCATCCACTACC }\end{array}$ & $\mathrm{C}$ & 57 \\
\hline D5S107 & $5 q 11.2-13.3$ & Unknown & $\begin{array}{l}\text { GATCCACTTTAACCCAAATAC } \\
\text { GGCATCAACTTGAACAGCAT }\end{array}$ & $\mathrm{F}$ & 53 \\
\hline D10S215 & $10 q 22-23$ & PTEN (nearby) & $\begin{array}{l}\text { TGGCATCATTCTGGGGA } \\
\text { GCTTTACGTTTCTTCACATGGT }\end{array}$ & $\mathrm{C}$ & 55 \\
\hline D10S2491 & $10 \mathrm{q} 23$ & PTEN (flanking Exon1) & $\begin{array}{l}\text { GTTAGATAGAGTACCTGCACTC } \\
\text { TTATAAGGACTGAGTGAGGGA }\end{array}$ & $\mathrm{F}$ & 50 \\
\hline D10S579 & $10 q 22-23$ & PTEN (nearby) & $\begin{array}{l}\text { CCGATCAATGAGGAGTGCC } \\
\text { ATACACCCAGCCAATGCTGC }\end{array}$ & $\mathrm{C}$ & 61 \\
\hline TH.PCR2 & $11 \mathrm{p} 15,5$ & Tyrosine hydroxylase (intragenic) & $\begin{array}{l}\text { CAGCTGCCCTAGTCAGCAC } \\
\text { GCTTCCGAGTGCAGGTCACA }\end{array}$ & $\mathrm{D}$ & 58 \\
\hline D11S528 & $11 q 23.3$ & MLL1 (nearby) & $\begin{array}{l}\text { AATGGTGTCCCCACACATGT } \\
\text { TCCTACCTACCGAGCTTAAA }\end{array}$ & $\mathrm{D}$ & 52 \\
\hline D13S1699 & $13 q 12-13$ & BRCA2 (nearby) & $\begin{array}{l}\text { AGACAGAGAATCTCAACTGG } \\
\text { TTTGATTTTCACAGCAGATG }\end{array}$ & $\mathrm{C}$ & 54 \\
\hline D17S855 & $17 q 21$ & BRCA1 (intragenic) & $\begin{array}{l}\text { GGATGGCCTTTTAGAAAGTGG } \\
\text { ACACAGACTTGTCCTACTGCC }\end{array}$ & $\mathrm{C}$ & 58 \\
\hline $\begin{array}{l}\text { D17S250 } \\
\text { (Mfd 15) }\end{array}$ & 17q11.2-12 & PNMT (nearby) & $\begin{array}{l}\text { GGAAGAATCAAATAGACAAT } \\
\text { GCTGGCCATATATATATTTAAACC }\end{array}$ & $\mathrm{F}$ & 53 \\
\hline TP53.PCR15 & 17p13.1 & p53 (intragenic) & $\begin{array}{l}\text { AGGGATACTATTCAGCCCGAGGTG } \\
\text { ACTGCCACTCCTTGCCCCATTC }\end{array}$ & $\mathrm{F}$ & 64 \\
\hline D18S59 & 18pter-p22 & Unknown & $\begin{array}{l}\text { AGCTTCTATCCAACAGGGGC } \\
\text { ACCAGAATGTGAACGACCCT }\end{array}$ & $\mathrm{D}$ & 54 \\
\hline
\end{tabular}

${ }^{\mathrm{a}}$ PCR Optimizer Kit (Invitrogen Corporation). 
D10S579 ${ }^{14}$ ); genes associated with poor prognosis (TP53.PCR15, ${ }^{15,16}$ TH.PCR2, ${ }^{17}$ D13S1699 ${ }^{18,19}$ ); genes involved in DNA mismatch repair (BAT26 related to $\mathrm{MSH} 2^{20}$ ); and genes used in previous microsatellite studies (D5S107 ${ }^{21,22}$ ), respectively.

The microsatellite loci were amplified by polymerase chain reaction (PCR) as published previously. ${ }^{22}$ The primer sequences were obtained from the 'Genome Data Base' at www.gdb.org and the primers were synthesized on a DNA synthesizer, Model 392, from Applied Biosystems Inc. (Foster City, CA, USA). The forward primers were endlabeled with Cy-5. The PCR products were separated on a $6 \%$ polyacrylamide gel and automatically detected on an ALF DNA Sequencer (Amersham Pharmacia Biotech). In each case duplicate assays were run for each microsatellite locus, and for some microsatellite loci a third run was performed to rule out spurious results for allelic loss.

Loss of heterozygosity (LOH) was defined as a complete or partial (at least 50\%) signal reduction of one of the two corresponding alleles in the matching tumor and recurrence DNA, respectively, compared to normal DNA. It was also analyzed whether the longer or the shorter allele was lost. Microsatellite instability (MSI) was defined as the presence of new alleles in the tumor or recurrence DNA, which were not present in normal DNA.

Tumors were considered to be genetically related, if genetic alterations ( $\mathrm{LOH}$ and MSI) were identical. Additional alterations in the later occurring tumor and the local recurrence, respectively, were consid- ered to be compatible with a genetic relationship according to a progression model with increase of genetic alterations during tumor growth and spread. ${ }^{20,23}$ If genetic alterations present in the earlier occurring tumor were not found in the later occurring tumor, a genetic relationship was not considered since an acquired genetic alteration is considered to be retained during tumor progression.

\section{Immunohistochemistry}

Immunohistochemistry for estrogen (ER) and progesterone receptors (PR) and HER-2/neu was performed on consecutive paraffin sections using standardized procedures on automated systems for ER and PR (Ventana Medical Systems Inc., AZ, USA) and for HER-2/neu (DAKO, Glostrup, Denmark). As primary antibodies clone 6F11 (Ventana) was used for ER and clone 1A6 (Ventana) for PR. The HercepTest $^{\mathrm{TM}}$ (DAKO) was used for HER-2/neu determination. Cases were scored as positive for ER and PR, respectively, when at least $10 \%$ of nuclei were immunoreactive. HER-2/neu was scored according to the manufacturer's instructions (DAKO). Score $3+$ was considered positive for HER-2/neu overexpression.

\section{Results}

The clinicopathological features of the cases studied are detailed in Figure 1. The median age of the eight

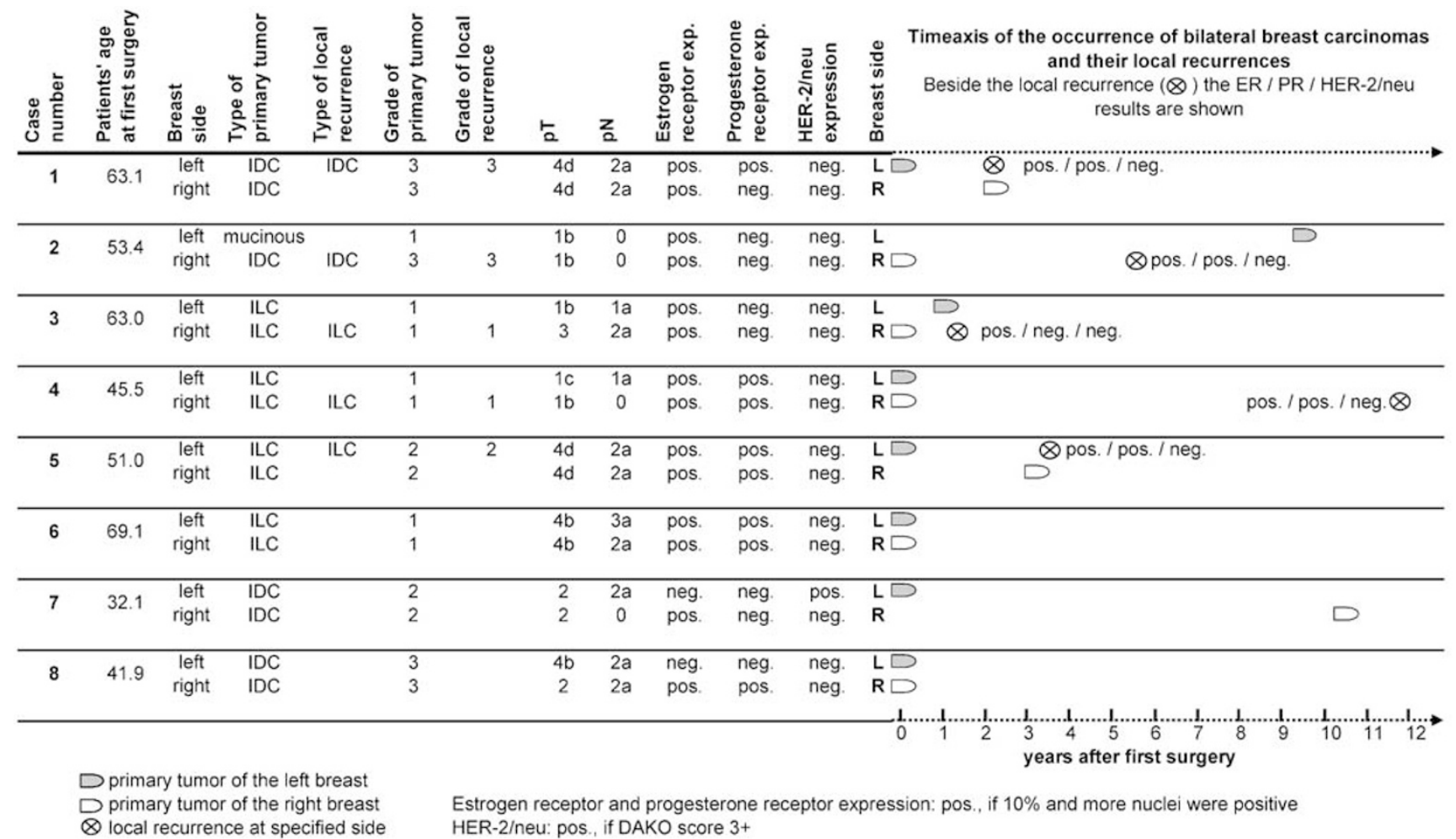

Figure 1 Clinicopathological details of the cases studied. 
patients at first surgery was 53.4 years (range: $32.1-$ 69.1 years). In three of the eight cases the bilateral breast carcinomas occurred synchronously. In five cases, the contralateral carcinoma occurred within a time period between 1.3 and 10.1 years (median 2.4 years). The recurrent breast carcinomas occurred 1.2-11.8 years (median 3.4 years) after the first surgery. In three cases the LR occurred almost at the same time as the contralateral tumor, in one case the recurrence occurred earlier than the contralateral tumor and in one case $\mathbf{1 1 . 8}$ years after synchronous bilateral breast carcinoma. Occurrence of primary and recurrent tumors is detailed in Figure 1. Two patients with LRs were treated by bilateral mastectomy, two by mastectomy on one side and lumpectomy on the other side and one by lumpectomy on both sides. Axillary lymph node resection was performed in all cases. Histologically, three patients presented with bilateral infiltrating duct carcinoma of no specific type (IDC), four with bilateral infiltrating lobular carcinoma (ILC), and one with an IDC on one side and a mucinous carcinoma on the other side. In this latter case the LR was an IDC occurring on the same side as the primary IDC. In the other four cases with LR, the same tumor type was found both in the bilateral tumors and the LR, but with slight histological differences regarding the amount of desmoplastic stroma and the percentage of solid formations. Thus, histological features could not determine a relationship.

Postoperative staging according to UICC guidelines showed varying tumor categories ranging from pT1b to pT4d. Bilateral lymph node metastases were present in five cases, unilateral in two cases, and one case was free of lymph node involvement. Tumor grades ranged from grade 1 to grade 3 and differed only in one case between the left- and the right-sided carcinoma. All LRs showed the same tumor grade as the ipsilateral carcinoma.

Three of eight bilateral breast carcinomas showed divergent results concerning ER, PR and HER-2/neu immunoreactivity. Four of the five LRs showed identical immunohistochemical results compared to the ipsilateral tumor. The immunohistochemical results are further detailed in Figure 1.

Of the investigated microsatellite loci, 180 revealed informative results, of which 13 harbored MSI and $37 \mathrm{LOH}$. In the primary tumors the most frequent genetic alterations were detected at D17S250 (six), followed by D18S59 (five). Alterations at a microsatellite locus related to BRCA1 (D17S855) were found in four out of eight bilateral breast carcinomas. Other microsatellite loci, particularly, those associated with BRCA2, p53 and PTEN did not show frequent genetic alterations.

Discrepancies between the bilateral primary tumors regarding the occurrence of $\mathrm{LOH}$ or MSI were found in all eight cases ranging from two to five discrepant loci per case (median 3). Details are shown in Figure 2. These discrepancies made it unlikely that the bilateral tumors were related. In four of the five cases with LR, all genetic alterations present in the ipsilateral primary tumor were found in the LR whereas the genetic alterations were discrepant to the contralateral tumor. In addition, one to two additional genetic alterations that were not present in the primary carcinomas were found in the LR. All genetic alterations present in the ipsilateral primaries were retained in any of the LR.

A discrepancy between the LR and the ipsilateral breast carcinoma but similarity to the contralateral primary was found in one case of an ILC. This case was also clinically distinctive from the other cases, since the LR appeared 11.8 years after the primary diagnosis and was associated with multiple bone metastases.

\section{Discussion}

Bilateral carcinomas of the breast with LR may be considered an experiment of nature for the study of tumor spread. They can potentially reflect (i) metastatic spread of one tumor; (ii) two tumors of which one recurred; or (iii) three unrelated neoplasms. In the clinical management of breast carcinoma patients, it is important to separate local recurrences of a previous ipsilateral breast carcinoma from metastases to the contralateral breast, since the former are considered a local process whereas the latter are considered to reflect systemic tumor spread. Molecular analysis on microdissected tumor tissue may help to uncover the genetic relationship of multiple, simultaneously or subsequently occurring tumors.

Although the number of cases was limited in this study, the results may be valuable for the understanding of multiple occurrence and spread of breast carcinoma. We consider three observations as being of further interest. First, in accordance with other studies, bilateral breast carcinomas are genetically unrelated. ${ }^{2-4}$ Second, like in unilateral invasive and noninvasive breast carcinomas, LR of breast carcinoma is most likely related to the preceding carcinoma of the ipsilateral breast and not a de novo occurring carcinoma. ${ }^{22,24}$ Third, like in unilateral breast carcinomas, LR in bilateral carcinomas is also characterized by an increase of genetic alterations.

In the particular setting of metachronous bilateral carcinoma of the breast, it is possible that both the recurrence and the contralateral tumor are metastatic. However, our findings suggest that usually subsequent carcinoma of the contralateral breast develops independently and not due to metastatic spread and that the LR is related to the preceding ipsilateral tumor.

The origin of the locally recurrent tumors, however, remains unclear. There are several possibilities that we previously discussed in detail. ${ }^{22}$ One possibility is the development of the LR from vital tumor cells derived from the ipsilateral tumor. At 


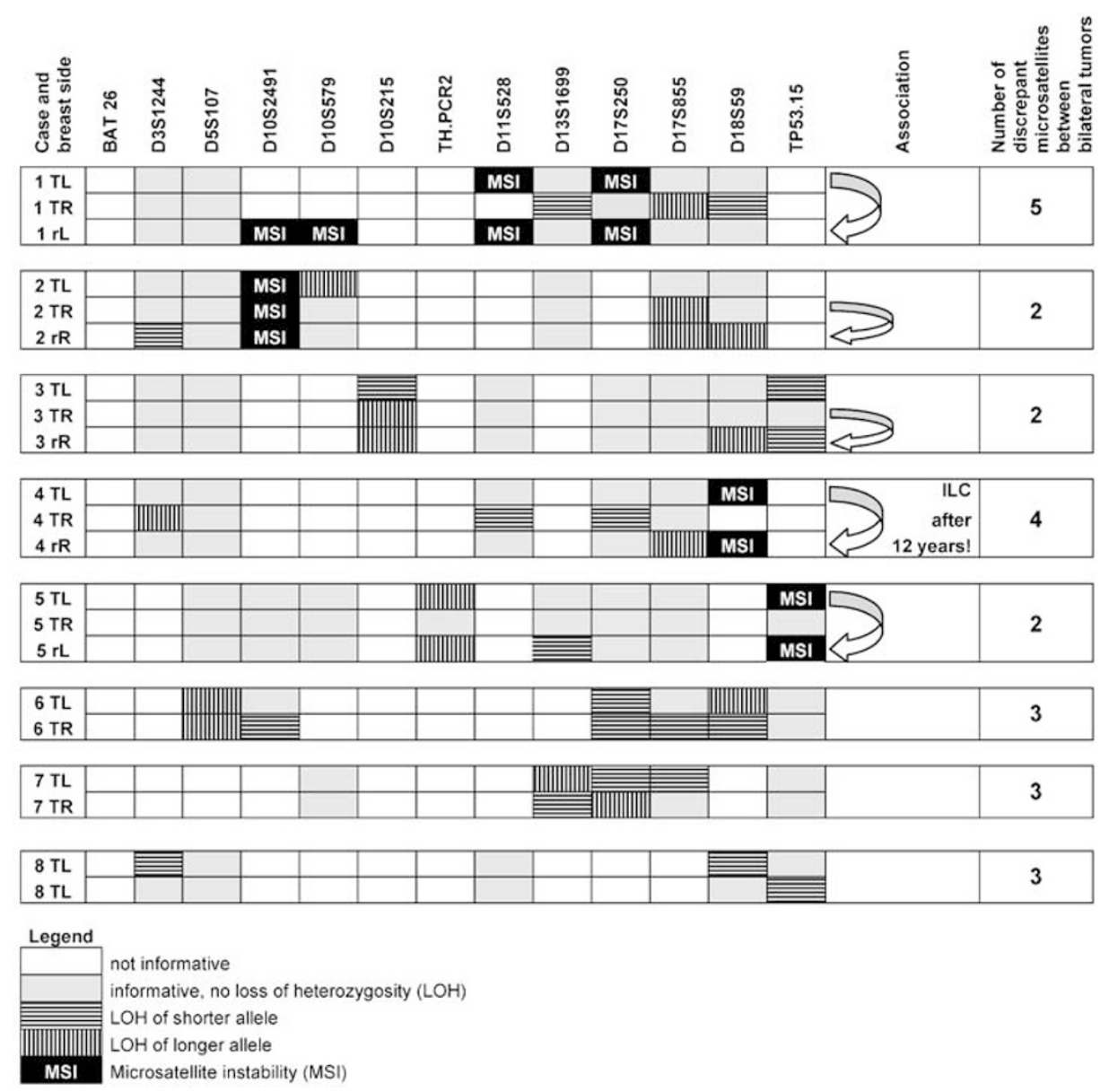

Figure 2 TR, tumor right; TL, tumor left; rR, local recurrence right; rL, local recurrence left; LOH, loss of heterozygosity; MSI, microsatellite instability; ILC, infiltrative lobular carcinoma.

least in a subset of cases, tumor cells may survive for many years in the area of the remaining 'tumor bed', but also in lymph vessels, even after surgical treatment, radiation and chemotherapy, and proliferate after a period of dormancy. Another possibility is the development from the same precursor lesion as the ipsilateral primary carcinoma, such as ductal carcinoma in situ, ductal hyperplasia with or without atypia or even from normally appearing yet already genetically modified ductal epithelium. ${ }^{24}$ In the setting of metastatic tumor spread, LRs may also originate from a contralateral tumor, as in our case 4.

To determine the genetic relationship of multiple tumors in one organ and system, several molecular methods can be used, in particular, X-chromosomebased methods like the HUMARA assay, sequence analysis of frequently mutated genes and the analysis of microsatellites. ${ }^{25}$ The advantage of microsatellite analysis is, in particular, its independence from patient's sex and the presence of specific mutations. Furthermore, microsatellite analysis can be performed on DNA extracted from formalin-fixed, paraffin-embedded tissue. By analysis of various microsatellites, a particular profile of alterations is obtained for each tumor that allows in most cases to assess the genetic relationship. It is assumed that once an allelic loss or MSI occurred the alteration is retained during tumor progression. ${ }^{20,23,26}$ Therefore, recurrent or metastatic tumors share the profile of genetic alterations of the tumor they originate from but due to an increase of genetic instability may contain additional alterations. In contrast, genetically unrelated tumors show a discordant profile of alterations that cannot be explained by an acquisition of additional alterations in the recurrent or metastatic tumor cell clones. Thus, a recurrent or metastatic tumor that does not retain genetic alterations found in the primary tumor can be considered genetically unrelated. ${ }^{27}$ Therefore, it can be assumed that only one discrepant microsatellite alteration is sufficient to rule out the genetic relationship of two neoplasms and a greater number of discrepant microsatellite alterations will more strongly support this assumption.

A direct comparison of most of the bilateral carcinomas and their local recurrences by its histological pattern and the expression of ER, PR and HER-2/neu did not allow to judge a potential relationship. In particular, a relationship of the bilateral and recurrent invasive lobular carcinomas 
could not be determined by light microscopy alone. Only in case 2 (Figure 1) it was possible to determine an association of the tumors by histology since only one carcinoma and its ipsilateral recurrence revealed the identical histological type and grade (IDC, grade 3), whereas on the contralateral side a mucinous carcinoma was diagnosed.

Although the number of cases in this study is small and, thus, the conclusions drawn are limited, we think that our findings have some practical implications. In particular, it might be useful in a subset of multiple tumors with similar histological pattern to determine whether a neoplasm has newly occurred or is locally recurrent or metastatic. This can be obtained easily and at affordable financial expenses by molecular analysis, for example, microsatellite analysis. The result whether a tumor is locally limited at low stage or widely spread at high stage is not of academic interest but has potential therapeutical consequences.

\section{Acknowledgement}

This study was supported by the Austrian Cancer Aid Styria (Project number: EF 05/00).

\section{References}

1 Chen Y, Thompson W, Semenciw R, et al. Epidemiology of contralateral breast cancer. Cancer Epidemiol Biomarkers Prev 1999;8:855-861.

2 Noguchi S, Motomura K, Inaji H, et al. Differentiation of primary and secondary breast cancer with clonal analysis. Surgery 1994;115:458-462.

3 Shibata A, Tsai YC, Press MF, et al. Clonal analysis of bilateral breast cancer. Clin Cancer Res 1996;2: $743-748$.

4 Imyanitov EN, Suspitsin EN, Grigoriev MY, et al. Concordance of allelic imbalance profiles in synchronous and metachronous bilateral breast carcinomas. Int J Cancer 2002;100:557-564.

5 Kollias J, Ellis IO, Elston CW, et al. Prognostic significance of synchronous and metachronous bilateral breast cancer. World J Surg 2001;25:1117-1124.

6 Elston CW, Ellis IO. Pathological prognostic factors in breast cancer. I. The value of histological grade in breast cancer: experience from a large study with longterm follow-up. Histopathology 1991;19:403-410.

7 Tavassoli FA. Pathology of the Breast. 2nd edn. Appleton Lange: Stanford, CT, 1998.

8 Sobin LH, Wittekind C. TNM Classification of Malignant Tumors - International Union Against Cancer (UICC). 6th edn. Wiley-Liss: New York, 2002.

9 Deng G, Lu Y, Zlotnikov G, et al. Loss of heterozygosity in normal tissue adjacent to breast carcinomas. Science 1996;274:2057-2059.

10 Kerangueven F, Eisinger F, Noguchi T, et al. Loss of heterozygosity in human breast carcinomas in the ataxia telangiectasia, Cowden disease and BRCA1 gene regions. Oncogene 1997;14:339-347.

11 Nagai MA, Yamamoto L, Salaorni S, et al. Detailed deletion mapping of chromosome segment 17q12-21 in sporadic breast tumours. Genes Chromosomes Cancer 1994;11:58-62.

12 Tran Y, Benbatoul K, Gorse K, et al. Novel regions of allelic deletion on chromosome 18p in tumors of the lung, brain and breast. Oncogene 1998;17:3499-3505.

13 Garcia JM, Silva JM, Dominguez G, et al. Allelic loss of the PTEN region (10q23) in breast carcinomas of poor pathophenotype. Breast Cancer Res Treat 1999;57: 237-243.

14 Shi W, Zhang X, Pintilie M, et al. Dysregulated PTEN$\mathrm{PKB}$ and negative receptor status in human breast cancer. Int J Cancer 2003;104:195-203.

15 Overgaard J, Yilmaz M, Guldberg P, et al. TP53 mutation is an independent prognostic marker for poor outcome in both node-negative and node-positive breast cancer. Acta Oncol 2000;39:327-333.

16 Jones $\mathrm{MH}$, Nakamura Y. Detection of loss of heterozygosity at the human TP53 locus using a dinucleotide repeat polymorphism. Genes Chromosomes Cancer 1992;5:89-90.

17 Winqvist R, Hampton GM, Mannermaa A, et al. Loss of heterozygosity for chromosome 11 in primary human breast tumors is associated with poor survival after metastasis. Cancer Res 1995;55:2660-2664.

18 Eiriksdottir G, Johannesdottir G, Ingvarsson S, et al. Mapping loss of heterozygosity at chromosome 13q: loss at 13q12-q13 is associated with breast tumour progression and poor prognosis. Eur J Cancer 1998;34:2076-2081.

19 van den Berg J, Johannsson O, Hakansson S, et al. Allelic loss at chromosome 13q12-q13 is associated with poor prognosis in familial and sporadic breast cancer. Br J Cancer 1996;74:1615-1619.

20 Loeb LA. Microsatellite instability: marker of a mutator phenotype in cancer. Cancer Res 1994;54:5059-5063.

21 Catasus L, Machin P, Matias-Guiu X, et al. Microsatellite instability in endometrial carcinomas: clinicopathologic correlations in a series of 42 cases. Hum Pathol 1998;29:1160-1164.

22 Regitnig P, Moser R, Thalhammer M, et al. Microsatellite analysis of breast carcinoma and corresponding local recurrences. J Pathol 2002;198:190-197.

23 Lengauer C, Kinzler KW, Vogelstein B. Genetic instabilities in human cancers. Nature 1998;396: 643-649.

24 Lininger RA, Fujii H, Man YG, et al. Comparison of loss of heterozygosity in primary and recurrent ductal carcinoma in situ of the breast. Mod Pathol 1998;11:1151-1159.

25 Diaz-Cano SJ, Blanes A, Wolfe HJ. PCR techniques for clonality assays. Diagn Mol Pathol 2001;10:24-33.

26 Pozo-Garcia L, Diaz-Cano SJ. Clonal origin and expansions in neoplasms: biologic and technical aspects must be considered together. Am J Pathol 2003;162: 353-354.

27 Tang M, Pires Y, Schultz M, et al. Microsatellite analysis of synchronous and metachronous tumors: a tool for double primary tumor and metastasis assessment. Diagn Mol Pathol 2003;12:151-159. 